\title{
A New Application of Hermite Collocation Method
}

\author{
Chandrali Baishya \\ Department of Studies and Research in Mathematics \\ Tumkur University, Tumkur -572103, Karnataka, India \\ E-mail: baishyachandrali@gmail.com \\ (Received August 29, 2018; Accepted October 31, 2018)
}

\begin{abstract}
This paper reflects the advantage of a new approach of using Hermite orthogonal basis elements to solve nonlinear differential equations. This method is based on a successive integration technique. To illustrate the method and to establish the efficiency of the method, it is applied to certain linear and nonlinear differential equations. The obtained numerical results show that the proposed method is a powerful numerical technique to solve nonlinear differential equations.
\end{abstract}

Keywords- Hermite polynomial basis, Collocation method, Nonlinear differential equations.

\section{Introduction}

In recent years, when the nature of differential equations become more and more sophisticated, collocation methods are treated as powerful tools to solve such equations. Various collocation methods have been examined by a number of Russian researchers in the case of linear integro-differential and differential equations. Russell and Shampin (1972); Boor and Swartz (1973) studied collocation with piecewise polynomials for nonlinear boundary value problems in ordinary differential equations. Boor and Swartz (1973) found optimal global estimates and established super convergence at certain points. Ronto (1971) utilized collocation based on polynomials for a system of first-order linear differential equations. Many authors depend on the Chebyshev collocation method to solve linear differential equations (Sezer and Kaynak, 1996; Akyüz and Sezer, 2003; Dolapci, 2004), integro-differential equations (Akyüz and Sezer, 1999), nonlinear differential equations (Akyüz-Daşcıog̈lu and Çerdik-Yaslan, 2011), partial differential equations (Keşan, 2003). Tau-collocation method (Lanczos, 1938; Liu, 1988; Sam and Liu, 2004) is another collocation method used by many researchers. Rawashdeh (2006) has applied the collocation spline method to solve a type of semi-differential equations. Even though Bernstein polynomial bases are not orthogonal, they are also used widely to solve many differential and integro-differential equations of physical importance (Bhattacharya and Mandal, 2008; Yousefi and Behroozifar, 2010). In Gürbüz et al. (2014), authors have applied the Laguerre collocation method to solve Fredholm integro-differential equations. Conte and Paternoster (2009) introduces multistep collocation methods for the numerical integration of Volterra Integral Equations, which depend on the numerical solution in a fixed number of previous time steps. In Funaro (1992), author devoted the chapters on analysis of approximate solution techniques, known as spectral collocation methods, for differential equations based on classical orthogonal polynomials.

Hermite polynomials are extensively used for numerical computation (Kalateh Bojdi et al., 2013; Brill, 2002; Dyksen and Lynch, 2000; Fazeli et al., 2012; Yalçinbaş et al., 2011). One of the advantages in using Hermite polynomials as a tool for functions expansion is that it gives a good representation of smooth functions provided that the function is infinitely differentiable (Andrews, 
1985; Dyksen and Lynch, 2000).

In this paper, we have applied the Hermite collocation method with a new approach of successive integration. Here we assume the derivative term as a series of Hermite orthogonal polynomials and then by successive integrations obtain the expressions for various terms present in the differential equation again in terms of Hermite polynomials. On substitution of these terms in the given equation and then using suitable collocation points, we obtain a system of equations.

\section{Description of the Proposed Method}

In this section, we have discussed the preliminaries of Hermite polynomial and the details of the proposed method to solve linear and nonlinear differential equations.

\subsection{Hermite Polynomials}

Definition 2.1 Hermite polynomial of order $n$ is denoted and defined by

where,

$$
H_{n}(x)=\sum_{r=0}^{\left[\frac{n}{2}\right]} \frac{(-1)^{r} n !}{r !(n-2 r) !}(2 x)^{n-2 r}
$$

$$
[\mathrm{n} / 2]=\left\{\begin{array}{l}
\frac{\mathrm{n}}{2}, \text { if } \mathrm{n} \text { is even } \\
\frac{\mathrm{n}-1}{2}, \text { if } \mathrm{n} \text { is odd }
\end{array}\right. \text {. }
$$

\section{Definition 2.2}

Rodrigues formula: Using the generating function, we obtain the Rodrigues formula as,

$$
H_{n}(x)=(-1)^{n} e^{x^{2}} \frac{d^{n}}{d x^{n}} e^{-x^{2}}
$$

\section{Definition 2.3}

Orthogonal Property

$$
\int_{-\infty}^{\infty} \mathrm{e}^{-\mathrm{x}^{2}} \mathrm{H}_{\mathrm{n}}(\mathrm{x}) \mathrm{H}_{\mathrm{m}}(\mathrm{x}) \mathrm{dx}=\left\{\begin{array}{ll}
0, & \text { if } \mathrm{m} \neq \mathrm{n} \\
\sqrt{\pi} 2^{\mathrm{n}} \mathrm{n} !, & \text { if } \mathrm{m}=\mathrm{n}
\end{array} .\right.
$$

\subsection{Hermite Collocation Method}

In this section, Hermite polynomial together with collocation method is used to solve the ordinary differential equation. Consider the general nth order ordinary differential equation is of the form

$$
y^{(n)}(t)=f\left(t, y, y^{\prime}, y^{\prime \prime}, \ldots y^{(n-1)}\right)
$$

With the initial conditions

$$
\mathrm{y}^{(\mathrm{i})}\left(\mathrm{t}_{0}\right)=\alpha_{\mathrm{i}}, \quad \mathrm{i}=0,1,2,3, \ldots(\mathrm{n}-1)
$$

where $\alpha_{\mathrm{i}}$ 's are constants.

Let us assume that,

$y^{(n)}(t) \approx B^{T} H(t)=\sum_{i=0}^{n} c_{i} H_{i}(t)$ 
International Journal of Mathematical, Engineering and Management Sciences

Vol. 4, No. 1, 182-190, 2019

https://dx.doi.org/10.33889/IJMEMS.2019.4.1-016

where,

$$
\begin{aligned}
& \mathrm{B}^{\mathrm{T}}=\left(\mathrm{c}_{0}, \mathrm{c}_{1}, \ldots . . \mathrm{c}_{\mathrm{n}}\right) \\
& \mathrm{H}(\mathrm{t})=\left(\mathrm{H}_{0}(\mathrm{t}), \ldots \ldots \mathrm{H}_{\mathrm{n}}(\mathrm{t})\right) .
\end{aligned}
$$

Now, Hermite polynomial coefficients to be determined. Integrating Eq (3) with respect to $t$ from $\mathrm{t}_{0}$ to $\mathrm{t}, \mathrm{n}$ times yields.

$$
\begin{aligned}
& y^{(n-1)}(t)=y\left(t_{0}\right)+\int_{t_{0}}^{t} B^{T} \quad H(t) \quad d t \\
& y^{(n-2)}(t)=y\left(t_{0}\right)+y^{\prime}\left(t_{0}\right)+\int_{t_{0}}^{t} \int_{t_{0}}^{t} B^{T} \quad H(t) \quad d t \\
& \text { : } \\
& y^{\prime}(t)=\sum_{i=0}^{n-1} y^{(i)}\left(t_{0}\right)+\int_{t_{0}}^{t} \int_{t_{0}}^{t} \ldots \int_{t_{0}}^{t} B^{T} \quad H(t) \quad d t \\
& y(t)=\sum_{i=0}^{n} y^{(i)}\left(t_{0}\right)+\int_{t_{0}}^{t} \int_{t_{0}}^{t} \ldots \int_{t_{0}}^{t} B^{T} \quad H(t) \quad d t
\end{aligned}
$$

Substituting Eqn. (4) in Eqn. (1) and collocating at the collocation points $t_{i}=\frac{i}{n}$, where $i=0,1, n$ and $n$ is any positive integer, we obtain a system of linear or nonlinear equations depending on the linearity or nonlinearity of the given equation 1 . Solving this system of equations we obtain the Hermite polynomial coefficients $\mathrm{B}^{\mathrm{T}}$ which yields the solution.

\section{Numerical Experiments}

Example 3.1 Consider the first order nonlinear ordinary differential equation

$\mathrm{y}^{\prime}=-\mathrm{y}^{2}$

With initial condition $\mathrm{y}(0)=1$.

The exact solution is $y(t)=\frac{1}{1+t}$.

Solution Let us assume the solution in the form,

$$
y^{\prime}(t)=\sum_{i=0}^{4} c_{i} H_{i}(t)
$$

This yields

$y^{\prime}(t)=c_{0}+c_{1} 2 t+c_{2}\left(4 t^{2}-2\right)+c_{3}\left(8 t^{3}-12 t\right)+c_{4}\left(16 t^{4}-48 t^{2}+12\right)$

Where $c_{i}, i=0,1, \ldots 4$, are Hermite polynomials coefficients to be determined. Applying the above-described procedure we obtain:

$y(t)=c_{4}\left(\frac{16 t^{5}}{5}-16 t^{3}+12 t\right)+c_{3}\left(2 t^{4}-6 t^{2}\right)+c_{2}\left(\frac{4 t^{3}}{3}-2 t\right)+c_{1} t^{2}+c_{0} t+1$ 
International Journal of Mathematical, Engineering and Management Sciences

Vol. 4, No. 1, 182-190, 2019

https://dx.doi.org/10.33889/IJMEMS.2019.4.1-016

Substituting Eqn. (6) and Eqn. (7) in Eqn. (5), we get

$$
\begin{aligned}
& c_{4}\left(16 t^{4}-48 t^{2}+12\right)+c_{3}\left(8 t^{3}-12 t\right)+c_{2}\left(4 t^{2}-2\right)+c_{1} 2 t+c_{0} \\
& +\left(c_{4}\left(\frac{16 t^{5}}{5}-16 t^{3}+12 t\right)+c_{3}\left(2 t^{4}-6 t^{2}\right)+c_{2}\left(\frac{4 t^{3}}{3}-2 t\right)+c_{1} t^{2}+c_{0} t+1\right)=0
\end{aligned}
$$

Collocating the obtained equation Eq (8) using following collocation points $\mathrm{t}_{0}=0, \mathrm{t}_{1}=\frac{1}{4}$, $\mathrm{t}_{2}=\frac{2}{4}, \mathrm{t}_{3}=\frac{3}{4}$ and $\mathrm{t}_{4}=1$, we get

$$
\begin{aligned}
& 12 \mathrm{c}_{4}-2 \mathrm{c}_{2}+\mathrm{c}_{0}+1=0, \\
& \left(\frac{881 \mathrm{c}_{4}}{320}-\frac{47 \mathrm{c}_{3}}{128}-\frac{23 \mathrm{c}_{2}}{48}+\frac{\mathrm{c}_{1}}{16}+\frac{\mathrm{c}_{0}}{4}+1\right)^{2}+\frac{145 \mathrm{c}_{4}}{16}-\frac{23 \mathrm{c}_{3}}{8}-\frac{7 \mathrm{c}_{2}}{4}+\frac{\mathrm{c}_{1}}{2}+\mathrm{c}_{0}=0, \\
& \left(\frac{41 \mathrm{c}_{4}}{10}-\frac{11 \mathrm{c}_{3}}{8}-\frac{5 \mathrm{c}_{2}}{6}+\frac{\mathrm{c}_{1}}{4}+\frac{\mathrm{c}_{0}}{2}+1\right)^{2}+\mathrm{c}_{4}-5 \mathrm{c}_{3}-\mathrm{c}_{2}+\mathrm{c}_{1}+\mathrm{c}_{0}=0, \\
& \left(\frac{963 \mathrm{c}_{4}}{320}-\frac{351 \mathrm{c}_{3}}{128}-\frac{15 \mathrm{c}_{2}}{16}+\frac{9 \mathrm{c}_{1}}{16}+\frac{3 \mathrm{c}_{0}}{4}+1\right)^{2}-\frac{159 \mathrm{c}_{4}}{16}-\frac{45 \mathrm{c}_{3}}{8}+\frac{\mathrm{c}_{2}}{4}+\frac{3 \mathrm{c}_{1}}{2}+\mathrm{c}_{0}=0, \\
& -20 \mathrm{c}_{4}+\left(-\frac{4 \mathrm{c}_{4}}{5}-4 \mathrm{c}_{3}-\frac{2 \mathrm{c}_{2}}{3}+\mathrm{c}_{1}+\mathrm{c}_{0}+1\right)^{2}-4 \mathrm{c}_{3}+2 \mathrm{c}_{2}+2 \mathrm{c}_{1}+\mathrm{c}_{0}=0 .
\end{aligned}
$$

Solving these we obtained the Hermite polynomial coefficients as $c_{4}=-0.034805220783117$, $\mathrm{c}_{3}=0.2215300759198, \quad \mathrm{c}_{2}=-1.019318388919264, \quad \mathrm{c}_{1}=2.299777282850779 \quad$ and $\mathrm{c}_{0}=-2.620974076983504$. Substituting these we obtain the solution of Eqn.(5) as

$$
\begin{aligned}
y(t)= & 1-0.03480\left(\frac{16 t^{5}}{5}-16 t^{3}+12 t\right)+0.22153\left(2 t^{4}-6 t^{2}\right)-1.019318\left(\frac{4 t^{3}}{3}-2 t\right) \\
& +2.29977728 t^{2}-2.62097 t
\end{aligned}
$$

Comparison between numerical and exact solutions are shown in Table 1.

Table 1. Numerical comparison of the exact solution and the solution obtained by the present method

\begin{tabular}{|c|c|c|}
\hline $\mathbf{t}$ & Numerical solution & Exact solution \\
\hline 2 & 0.833379477 & 0.8333333333 \\
\hline 0.4 & 0.714256065 & 0.714285714 \\
\hline 0.6 & 0.625097978 & 0.625000000 \\
\hline 0.8 & 0.555533211 & 0.555555555 \\
\hline 1.0 & 0.500072671 & 0.500000000 \\
\hline
\end{tabular}


International Journal of Mathematical, Engineering and Management Sciences

Vol. 4, No. 1, 182-190, 2019

https://dx.doi.org/10.33889/IJMEMS.2019.4.1-016

Example 3.2 Consider the third order linear ordinary differential equation

$$
\mathrm{y}^{(3)}+3 \mathrm{y}^{\prime \prime}+3 \mathrm{y}^{\prime}+\mathrm{y}=0
$$

With initial conditions $\mathrm{y}(0)=7, \quad \mathrm{y}^{\prime}(0)=-7$ and $\mathrm{y}^{\prime \prime}(0)=11$

The exact solution is $y(t)=7 \mathrm{e}^{-t}+2 t^{2} e^{-t}$

Solution Let us assume the approximate solution in terms of Hermite polynomial as

$$
y^{\prime \prime \prime}(t)=\sum_{i=0}^{3} c_{i} H_{i}(t)
$$

This gives

$$
\mathrm{y}^{\prime \prime \prime}(\mathrm{t})=\mathrm{c}_{0}+\mathrm{c}_{1} 2 \mathrm{t}+\mathrm{c}_{2}\left(4 \mathrm{t}^{2}-2\right)+\mathrm{c}_{3}\left(8 \mathrm{t}^{3}-12 \mathrm{t}\right)
$$

Substituting (11) and the expressions obtained after successive integration, in (9) we get

$$
\begin{aligned}
& 3\left(c_{3}\left(2 t^{4}-6 t^{2}\right)+c_{2}\left(\frac{4 t^{3}}{3}-2 t\right)+c_{1} t^{2}+c_{0} t+11\right)+\frac{c_{1} t^{4}}{12}+c_{3}\left(8 t^{3}-12 t\right)+c_{3}\left(\frac{t^{6}}{15}-\frac{t^{4}}{2}\right)+c_{2}\left(\frac{t^{5}}{15}-\frac{t^{3}}{3}\right) \\
& +3\left(c_{3}\left(\frac{2 t^{5}}{5}-2 t^{3}\right)+c_{2}\left(\frac{t^{4}}{3}-t^{2}\right)+\frac{c_{1} t^{3}}{3}+\frac{c_{0} t^{2}}{2}+11 t-7\right)+\frac{c_{0} t^{3}}{6}+c_{2}\left(4 t^{2}-2\right)+\frac{11 t^{2}}{2}+2 c_{1} t-7 t+c_{0}+7=0 .
\end{aligned}
$$

Now collocate the above equation using following collocation points

$\mathrm{t}_{0}=0, \mathrm{t}_{1}=\frac{1}{3}, \mathrm{t}_{2}=\frac{2}{3}, \mathrm{t}_{3}=1$, then we get

$-2 \mathrm{c}_{2}+\mathrm{c}_{0}+19=0$,

$-\frac{1}{43740}\left(256010 c_{3}+163608 c_{2}-45405 c_{1}-95040 c_{0}-1236870\right)=0$,

$-\frac{1}{4370}\left(256010 c_{3}+163608 c_{2}-45405 c_{1}-95040 c_{0}-1236870\right)=0$

$-\frac{1}{4370}\left(256010 c_{3}+163608 c_{2}-45405 c_{1}-95040 c_{0}-1236870\right)=0$.

Solving this system of equations, we get Hermite polynomial coefficients as $c_{0}=-29.441666$, $c_{1}=19.78715, c_{2}=-5.220833$ and $c_{3}=0.7473913$. These in tern yield the solution of (9) as

$$
\begin{aligned}
y(t)= & 0.7473913\left(\frac{\mathrm{t}^{6}}{15}-\frac{\mathrm{t}^{4}}{2}\right)-5.220833\left(\frac{\mathrm{t}^{5}}{15}-\frac{\mathrm{t}^{3}}{3}\right)+1.648929166666667 \mathrm{t}^{4} \\
& -4.906944333333334 \mathrm{t}^{3}+\frac{11 \mathrm{t}^{2}}{2}-7 \mathrm{t}+7
\end{aligned}
$$

Table 2 gives a comparison of solution obtained by present method and exact solution. 
International Journal of Mathematical, Engineering and Management Sciences

Vol. 4, No. 1, 182-190, 2019

https://dx.doi.org/10.33889/IJMEMS.2019.4.1-016

Table 2. Numerical comparison of the exact solution and the present method

\begin{tabular}{|c|c|c|}
\hline $\mathrm{t}$ & Numerical solution & Exact solution \\
\hline 0.2 & 5.79659885 & 5.796613731 \\
\hline 0.4 & 4.906619309 & 4.906742736 \\
\hline 0.6 & 4.236530149 & 4.236825830 \\
\hline 0.8 & 3.720013081 & 3.720443822 \\
\hline 1.0 & 3.3103374081 & 3.3109149711 \\
\hline
\end{tabular}

Example 3.3 Lotka-Volterra equations, also known as predator-prey equations, are a pair of first-order nonlinear differential equations, frequently used to describe the dynamics of biological systems in which two species interact, one as a predator and the other as prey. The populations change through time according to the pair of equations

$\frac{d x}{d t}=\alpha x-\beta x y ; \frac{d y}{d t}=-\gamma x+\delta x y$

with initial condition $\mathrm{x}(0)=\mathrm{x}_{0}, \mathrm{y}(0)=\mathrm{y}_{0}$, where $\mathrm{x}$ is the prey population, $\mathrm{y}$ is the predator population, $\alpha$ is the natural growth rate of prey in the predation absence, $\beta$ is the predator's effect on the prey-population, $\gamma$ is the natural predator's death rate in the absence of prey to eat and $\delta$ is The prey's effect on the predator-population.

Solution Let us assume that solution in terms of Hermite polynomial as,

$x^{\prime}(t)=\sum_{i=0}^{5} c_{i} H_{i}(t)$

$\mathrm{y}^{\prime}(\mathrm{t})=\sum_{\mathrm{i}=0}^{5} \mathrm{~b}_{\mathrm{i}} \mathrm{H}_{\mathrm{i}}(\mathrm{t})$

On integration it gives

$x(t)=\frac{16 c_{5} t^{6}}{3}+\frac{16 c_{4} t^{5}}{5}+2 c_{3} t^{4}-40 c_{5} t^{4}+\frac{4 c_{2} t^{3}}{3}-16 c_{4} t^{3}+c_{1} t^{2}-6 c_{3} t^{2}+60 c_{5} t^{2}+c_{0} t-2 c_{2} t+12$

$y(t)=\frac{16 b_{5} t^{6}}{3}+\frac{16 b_{4} t^{5}}{5}+2 b_{3} t^{4}-40 b_{5} t^{4}+\frac{4 b_{2} t^{3}}{3}-16 b_{4} t^{3}+b_{1} t^{2}-6 b_{3} t^{2}+60 b_{5} t^{2}+b_{0} t-2 b_{2} t+12 b_{4} t+9$

Substituting (13) to (16) in (12) and using the collocation points $t_{i}=\frac{i}{4}, \quad i=0(1) 4$, we obtain a system of eight equations. For numerical results we consider initial condition as $x(0)=4, y(0)=9$ and the values of the parameters $\alpha=0.1, \beta=0.0014, \gamma=0.08, \delta=0.0012$. This yields the acceptable values as follows:

$\mathrm{c}_{0}=0.350441 ; \mathrm{c}_{1}=0.0172145 ; \mathrm{c}_{2}=0.000420786 ; \mathrm{c}_{3}=6.996557110825257 \times 10^{-6}$;

$\mathrm{c}_{4}=8.514562757418547 \times 10^{-8} ; \mathrm{c}_{5}=8.859331033843632 \times 10^{-10}$;

$\mathrm{b}_{0}=-0.677878 ; \mathrm{b}_{1}=0.0273787 ; \mathrm{b}_{2}=-0.000539367$;

$b_{3}=07.206093098874797 \times 10^{-6} ; b_{4}=-7.019638609809134 \times 10^{-8} ; b_{5}=0$. 
International Journal of Mathematical, Engineering and Management Sciences

Vol. 4, No. 1, 182-190, 2019

https://dx.doi.org/10.33889/IJMEMS.2019.4.1-016

A comparative analysis of the results obtained by exact solution, the proposed method and Laplace Adomian Decomposition method(LADM) (Paul et al., 2016) is given Table 3.

Table 3. Numerical comparison of exact solution and present method when $\mathrm{x}(0)=4, \mathrm{y}(4)=9, \alpha=0.1, \beta=0.0014, \gamma=0.08$ and $\delta=0.0012$

\begin{tabular}{|l|c|c|c|c|c|c|}
\hline & \multicolumn{2}{|c|}{ Exact } & \multicolumn{2}{c|}{ Hermite } & \multicolumn{2}{c|}{ LADM } \\
\hline $\mathbf{t}$ & $\mathbf{x}(\mathbf{t})$ & $\mathbf{y}(\mathbf{t})$ & $\mathbf{x}(\mathbf{t})$ & $\mathbf{x}(\mathbf{t})$ & $\mathbf{y}(\mathbf{t})$ \\
\hline 1. & 4.36734648 & 8.34983166 & 4.36734648 & 8.34983164 & 4.36423471 & 8.34470435 \\
\hline 2. & 4.77260007 & 7.75022106 & 4.77260006 & 7.75022109 & 4.76588351 & 7.74074456 \\
\hline 3. & 5.21966481 & 7.19734593 & 5.21966476 & 7.19734530 & 5.20875110 & 7.18416528 \\
\hline 4. & 5.71285281 & 6.6876798 & 5.71285233 & 6.68767319 & 5.69703097 & 6.67132682 \\
\hline 5. & 6.25692620 & 6.2179702 & 6.25692347 & 6.21793873 & 6.23534323 & 6.19887998 \\
\hline 6. & 6.85714350 & 5.78521821 & 6.85713245 & 5.78511393 & 6.82877602 & 5.76374307 \\
\hline 7. & 7.51931075 & 5.38665958 & 7.51927506 & 5.38638194 & 7.48293069 & 5.36308097 \\
\hline 8. & 8.24983784 & 5.01974814 & 8.24974008 & 5.01911007 & 8.20397089 & 4.99428600 \\
\hline 9. & 9.05580068 & 4.68213978 & 9.05556406 & 4.68082281 & 8.99867598 & 4.65496056 \\
\hline 10. & 9.94500969 & 4.37167823 & 9.94448951 & 4.36917493 & 9.87449896 & 4.34290125 \\
\hline 11. & 10.92608546 & 4.08638201 & 10.92502657 & 4.08192446 & 10.83962917 & 4.05608454 \\
\hline 12. & 12.00854112 & 3.82443260 & 12.00651797 & 3.81690581 & 11.90305989 & 3.79265371 \\
\hline 13. & 13.20287427 & 3.58416389 & 13.19920745 & 3.57200274 & 13.07466132 & 3.55090713 \\
\hline 14. & 14.52066904 & 3.36405263 & 14.51431162 & 3.34512145 & 14.36525868 & 3.32928773 \\
\hline 15. & 15.97470369 & 3.16271017 & 15.96409515 & 3.13416361 & 15.78671596 & 3.12637363 \\
\hline
\end{tabular}

\section{Conclusion}

This paper introduces a different approach to solving nonlinear differential equations using Hermite orthogonal basis elements. It is observed that this numerical technique has a high accuracy rate while compared with the exact solution or solutions obtained by other methods. In Example 3.3 it is seen that the present method gives a better approximation for a system of nonlinear differential equations compared to LADM. In the future, there is a scope of using the method for solving fractional differential equations.

\section{Conflict of Interest}

I confirm that this article contents have no conflict of interest.

\section{Acknowledgment}

I would like to thank the reviewers for their constructive comments. 
International Journal of Mathematical, Engineering and Management Sciences

Vol. 4, No. 1, 182-190, 2019

https://dx.doi.org/10.33889/IJMEMS.2019.4.1-016

\section{References}

Akyüz, A., \& Sezer, M. (1999). A Chebyshev collocation method for the solution of linear integro-differential equations. International journal of computer mathematics, 72(4), 491-507.

Akyüz, A., \& Sezer, M. (2003). Chebyshev polynomial solutions of systems of high-order linear differential equations with variable coefficients. Applied Mathematics and Computation, 144(2-3), 237-247.

Andrews, L. C. (1985). Special functions for engineers and applied mathematicians, Macmillan publishing company, New York.

Bhattacharya, S., \& Mandal, B. N. (2008). Use of Bernstein polynomials in numerical solution of Volterra integral equations, Applied Mathematical Science, 2(36), 1773-1787.

Boor, C. D., \& Swartz, B. (1973). Collocation at Gaussian points, SIAM Journal on Numerical Analysis, 10, 582-606.

Brill, S. H. (2002). Analytical solution of Hermite collocation discretization of the Steady-State Convection Diffusion equation, International Journal of Differential Equations and Applications, 4, 141-155.

Conte, D., \& Paternoster, B. (2009). Multistep collocation methods for Volterra Integral Equations, Applied Numerical Mathematics. 59(8), 1721-1736.

Dolapci, I. T. (2004). Chebyshev collocation method for solving linear differential equations, Mathematical \& Computational Applications, 9(1), 107-115.

Dyksen, W. R., \& Lynch, R. E. (2000). A new decoupling technique for the Hermite cubic collocation equations arising from boundary value problems, Mathematics and Computers in Simulation, 54(4-5), 359-372.

Fazeli, S., Hojjati, G., \& Shahmorad, S. (2012). Multistep Hermite collocation methods for solving Volterra integral equations. Numerical Algorithms, 60(1), 27-50.

Funaro, D. (1992). Polynomial approximations of differential equation. Journal of Mathematical Physics, 17 , $123-199$.

Gürbüz, B., Sezer, M., \& Güler, C. (2014). Laguerre collocation method for solving Fredholm integro-differential equations with functional arguments. Journal of Applied Mathematics, 2014.

Kalateh Bojdi, Z., Ahmadi-Asl, S., \& Aminataei, A. (2013). Operational matrices with respect to Hermite polynomials and their applications in solving linear differential equations with variable coefficients. Journal of Linear and Topological Algebra, 2(2), 91-103.

Keşan, C. (2003). Chebyshev polynomial solutions of second-order linear partial differential equations. Applied Mathematics and Computation, 134(1), 109-124.

Lanczos, C. (1938). Trigonometric interpolation of empirical and analytical functions. Journal of Mathematics and Physics, 17(1-4), 123-199.

Liu, K. M. (1988). Numerical solution of differential eigenvalue problems with variable coefficients with the Tau-Collocation method. Mathematical and Computer Modelling, 11, 672-675.

Paul, S., Mondal, S. P., \& Bhattacharya, P. (2016). Numerical solution of Lotka Volterra prey predator model by using Runge-Kutta-Fehlberg method and Laplace Adomian decomposition method, Alexandria Engineering Journal, 55(1), 613-617.

Rawashdeh, E. A. (2006). Numerical solution of semidifferential equations by collocation method. Applied Mathematics and Computation, 174(2), 869-876.

Ronto, N. I. (1971). Application of the method of collocation to solve boundary value problems, Ukrainian Mathematical Journal., 23, 415-421. 
International Journal of Mathematical, Engineering and Management Sciences

Vol. 4, No. 1, 182-190, 2019

https://dx.doi.org/10.33889/IJMEMS.2019.4.1-016

Russell, R. D., \& Shampine, L. F. (1972). A collocation method for boundary value problems. Numerische Mathematik, 19(1), 1-28.

Sam, C. N., \& Liu, K. M. (2004). Numerical solution of partial differential equations with the Tau-Collocation Method.(Thesis). City University of Hong Kong, China.

Sezer, M., \& Kaynak, M. (1996). Chebyshev polynomial solutions of linear differential equations, International Journal of Mathematical Education in Science and Technology, 27(4), 607-611.

Yalçinbaş, S., Aynigül, M., \& Sezer, M. (2011). A collocation method using Hermite polynomials for approximate solution of pantograph equations, Journal of the Franklin Institute, 348(6), 1128-1139.

Yousefi, S. A., \& Behroozifar, M. (2010). Operational matrices of Bernstein polynomials and their applications. International Journal of Systems Science , 41(6), 709-716. 